\title{
Ultrasound-Enhanced Reactive Oxygen Species Responsive Charge-Reversal Polymeric Nanocarriers for Efficient Pancreatic Cancer Gene Delivery
}

Cong Zhang ${ }^{1,2 \#, ~ J i f a n ~ C h e n ~ 1,2 \#, ~ Y u e ~ S o n g ~}{ }^{1,2 \#, ~ J i a l i ~ L u o ~}{ }^{1,2}$, Peile Jin ${ }^{1,2}$, Xue Wang ${ }^{1,2}$, Lei Xin ${ }^{1,2}$, Fuqiang Qiu ${ }^{1,2}$, Jianting Yao ${ }^{1,2}$, Guowei Wang ${ }^{1,2,3^{*}}$, and Pintong Huang ${ }^{1,2^{*}}$

1 Department of Ultrasound in Medicine, The Second Affiliated Hospital of Zhejiang University School of Medicine, Zhejiang University, Hangzhou 310009, China.

2 Research Center of Ultrasound in Medicine and Biomedical Engineering, The Second Affiliated Hospital of Zhejiang University School of Medicine, Zhejiang University, Hangzhou 310009, China.

3 Hangzhou Global Scientific and Technological Innovation Center, Zhejiang University, Hangzhou 311200, China.

\# There author contributes equally.

*Corresponding authors, Email: huangpintong@zju.edu.cn,wangguowei@zju.edu.cn. 
Materials. The key materials were obtained from the following sources: branched polyethylenimine (PEI $25 \mathrm{KDa}$ ) and 2-(N,N-Diethylamino)ethyl acrylate (DEAEA) were purchased from Sigma-Aldrich (Shanghai, China). Azodiisobutyronitrile (AIBN) was purchased from Aladdin (Shanghai, China). 4-(Bromomethyl)phenylboronic acid was purchased from Soochiral Chemical Science \& Technology Co., Ltd (Suzhou, China). 1,2-Dioleoyl-sn-glycero-3-phosphoethanolamine (DOPE) were purchased from Avanti Polar Lipids Company (Alabaster, AL, USA). Cholesteryl hemisuccinate (CHEMS) was purchased from Tokyo Chemical Industry Co., Ltd (Shanghai, China). 1,2-Distearoyl-sn-glycero-3-phosphoethanol- amine $\mathrm{N}$-[methoxy(polyethylene glycol)-2000] (DSPE-PEG) was purchased from Laysan Bio. Inc (USA). 2',7'Dichlorofluorescin diacetateand and IR780 iodide was purchased from Sigma-Aldrich Co., Ltd (German).

Synthesis of B-PDEAEA. The B-PDEAEA was synthesized following the method proposed in previous literature. ${ }^{1}$ In short, AIBN (0.048 g, $\left.0.029 \mathrm{mmol}\right)$ and DEAEA (5 g, $29 \mathrm{mmol}$ ) were added to a $25 \mathrm{~mL}$ flask, deoxygenated with nitrogen at room temperature, and then polymerized at $65{ }^{\circ} \mathrm{C}$ for 24 hours. The viscous liquid was dissolved in $\mathrm{CH}_{2} \mathrm{Cl}_{2}$ and precipitate in cold n-hexame to obtain the PDEAEA, which was subsequently purified by reprecipitation three times and dried under a vacuum. The PDEAEA (0.3 g, 1.8 mmol tertiary amines) and 4-(bromomethyl)phenylboronic acid $(0.56 \mathrm{~g}, 2.6 \mathrm{mmol})$ were reacted in $20 \mathrm{~mL}$ DMF at room temperature for 24 hours. 
Using a dialysis bag (molecular weight cut-off: $3500 \mathrm{Da}$ ), the resulting solution was dialyzed against deionized water overnight and lyophilized to obtain B-PDEAEA $(0.5$ g, yield $92 \%$ as report).

Characterization of Polyplexes. Dissolve B-PDEAEA/TRAIL@IR780-liposomes, B-PDEAEA/Plasmid@Liposomes, Liposome, and IR780 load Liposome in HEPES buffer solution $(10 \mathrm{mM}, \mathrm{pH}=7.4)$. The size and zeta potential of the polyplexes were measured at $25{ }^{\circ} \mathrm{C}$ using a Zetasizer Nano-ZS (Malvern Instruments, UK). Data are presented as the mean $\pm \mathrm{SD}(\mathrm{n}=3)$. The morphology of polyplexes stained with uranyl acetate were investigated using TEM (JEM-1200EX). UV-vis spectrophotometer (SpectraMax M5, USA) was employed to collect the absorption spectra of the BTIL, BTL NPs, Lip, IR780 and IL.

B-PDEAEA Dissociation under Oxidative Condition. Dissolve B-PDEAEA in $1 \mathrm{mM}$ $\mathrm{H}_{2} \mathrm{O}_{2}$ at a concentration of $1 \mathrm{mM}$ at $37^{\circ} \mathrm{C}$. After $1 \mathrm{~h}, \mathrm{HPLC}$ was performed on $20 \mu \mathrm{L}$ samples of the solution. The HPLC system included a 1525 binary HPLC pump, a 2475 multi- $\lambda$-fluorescence detector and a 2998 photodiode array detector. In addition, a SunFireTM C18 column (Waters) $(4.6 \times 250 \mathrm{~mm}, 5 \mu \mathrm{m})$ was used to monitor the release of $p$-hydroxylmethylenephenol (HMP), a 15\% methanol aqueous solution was used as the eluent, and the flow rate was $1.0 \mathrm{~mL} / \mathrm{min}$. Introduce pure HMP as a standard at 10 min.

Cell Culture and Gene Transfection. BxPC-3 and AsPC-1 were purchased from Shanghai Cellular Institute of China Scientific Academy. All cell lines were maintained 
in RPMI 1640 (Hyclone) and added with 10\% heat-inactivated fetal bovine serum (BI), penicillin (100 units/mL) and streptomycin $(100 \mu \mathrm{g} / \mathrm{mL})$ in a humidified atmosphere of $5 \% \mathrm{CO}_{2}$ at $37{ }^{\circ} \mathrm{C}$. For luciferase gene transfection, cells were cultured in 48 -well plates ( $5 \times 10^{4}$ cells/well) and incubated overnight. The cells were treated with polyplexes at a dose of $1 \mu \mathrm{g}$ pLuci per well in fresh medium ( $0 \%$ or $10 \%$ FBS) for $4 \mathrm{~h}$. Then replaced the medium with $10 \%$ FBS medium. Finally, the luciferase gene expression was subsequently determined based on the standard protocol described in the manufacture manual (Promega) after $48 \mathrm{~h}$ of incubation.

Cellular Uptake and Intracellular Trafficking Study. BxPC-3 cells ( 3 x $10^{5}$ cells/well) were placed in 6-well plates and cultured for $24 \mathrm{~h}$. Then, $1 \mathrm{~mL}$ of medium containing BTIL (20 $\mu \mathrm{g} / \mathrm{mL})$ was replaced in the 6-well plates, and the cells were incubated for 2 , $4,6,12,24 \mathrm{~h}$, respectively. Next, the IR780 fluorescence intensity of $1 \times 10^{4}$ cells was analyzed by FCM to detect the uptake of BTIL complex at different time points.

To visualize the uptake of BTIL polyplexes, liposome was labeled with Cy5.5 to represent BTIL. After incubating for 12 hours, to label lysosomes, the cells were cultured with LysoTracker Green (Invitrogen, Thermo Fisher Scientific) at $200 \mathrm{nM}$ for $20 \mathrm{~min}$, and then DAPI ( $5 \mu \mathrm{g} / \mathrm{mL})$ was used to satin the nuclei for $5 \mathrm{~min}$. The cells were fixed with 4\% PFA fix solution and washed with PBS thrice before being imaged by CLSM. In addition, according to the manufacturer's instructions, Cy3 (Mirus Bio) and Cy5 (Mirus Bio) were used to label DNA to visualize the dissociation of BTIL polyplexes. BxPC-3 cells were placed on a glass bottom culture dish at a density of $5 \mathrm{x}$ 
$10^{4}$ cells per dish and cultured in $1 \mathrm{~mL}$ medium for $24 \mathrm{~h}$. Replaced the medium with 1 $\mathrm{mL}$ fresh medium containing BTIL polyplexes $(20 \mu \mathrm{g} / \mathrm{mL})$ with $0 \%$ FBS. After incubating for 4 hours, change the medium to a cell culture medium containing $10 \%$ FBS, and incubate the cells for another 24 hours. Then, before fixing with $4 \%$ PFA, cells were further treated with or without US $\left(1 \mathrm{MHz}, 0.2 \mathrm{~W} / \mathrm{cm}^{2}, 1 \mathrm{~min}\right)$. DAPI was used to stain the nuclei and CLSM was used to acquire images. The excitation wavelength of DAPI was $405 \mathrm{~nm}$.

In Vivo FL Imaging and Biodistribution of BTIL. In vivo FL imaging was performed using a Caliper IVIS Lumina II (Caliper Life Sciences, USA). The tumor-bearing mice were injected with BTIL solution (100 $\mu \mathrm{L}$, containing $20 \mu \mathrm{g}$ DNA) through the tail vein. Images were obtained at 1, 2, 4, 6, 8 and 12 hours after injection. Calculate and record the average FL intensities of the tumor sites via the IVIS Spectrum Software. Eventually, collect the tumors and major organs for ex vivo FL imaging.

Blood Clearance. $100 \mu \mathrm{L}$ of the BTIL solution was injected intravenously into tumorbearing mice. Blood collected from the orbit at time intervals was stored in Eppendorf tubes that have been flushed with heparin solution $(1 \mathrm{mg} / \mathrm{mL})$. The plasma was separated from the blood by centrifugation at $5000 \mathrm{rpm}$ for $10 \mathrm{~min}$ at $4{ }^{\circ} \mathrm{C}$, and then 50 $\mu \mathrm{L}$ of plasma was diluted to $100 \mu \mathrm{L}$ with PBS. The samples were measured using UVvis spectrophotometer. Use Prism Software to quantify the percentage of injection dose to the corresponding compound concentration. 
In vivo Toxicity Assay. The tumor-bearing mice were divided into two groups $(\mathrm{n}=$ 3). Mice were injected intravenously with $100 \mu \mathrm{L}$ of PBS as control, and the BTIL (6 $\mathrm{mg} / \mathrm{kg}$ ) injection group was taken as the treat group. The blood samples of each group were taken for analysis of blood biochemical index after 14 days treatment.

Stability of BTIL lipopolyplexes. The stability of BTIL lipopolyplexes was evaluated by measuring its size and zeta potential in HEPES and cell culture medium containing 10\% FBS during $48 \mathrm{~h}$ via particle analyzer (Nano-ZS, Malvern, England).

The encapsulation efficacy of IR780 and TRAIL. IR780 concentration $\left(c_{1}\right)$ was calculated from a standard curve (obtained by evaluating the absorbance of known concentrations of IR780 at $780 \mathrm{~nm}$ ) using UV-vis spectrometry (Tecan Spark, Tecan, Switzerland). The mass of encapsulated IR780 $\left(\mathrm{m}_{1}\right)$ encapsulation efficiency of IR780 was calculated as follows.

$$
\mathrm{m}_{1}=\mathrm{m}_{\text {initial }}-\mathrm{c}_{1} * \mathrm{v}_{\text {supernatant }}
$$

Loading efficiency of IR780 $=\mathrm{m}_{1} / \mathrm{m}_{\text {initial }}$

Where $\mathrm{v}_{\text {supernatant }}$ represents the volume of the supernatant and $\mathrm{m}_{\text {initial }}$ represents the initial mass of IR780.

Encapsulation efficiency of pDNA was measured using the NanoDrop (Thermo, Scientific) according to Elsana's paper. ${ }^{2}$

Western blot. To determine the expression of TRAIL, tumor tissues at different groups were lysed and protein contents were detected using BCA protein assay 
(Beyotime, China). Total proteins $(40 \mu \mathrm{g})$ were loaded and separated using 10\% SDSPAGE and then transferred onto a PVDF membrane (Millipore). The membrane was blocked with $3 \%$ BSA for $1 \mathrm{~h}$ and then incubated with the primary antibody against TRAIL (3219; Cell Signaling Technology, Inc.). The membrane was then rinsed in TBST and incubated with goat anti-rabbit antibodies at 1:5000. Finally, the membrane was rinsed and visualized with electrochemiluminescence detection reagent (Absin, Shanghai, China).

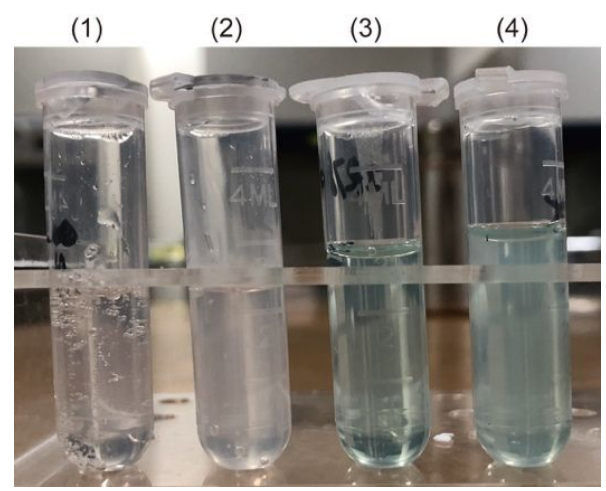

Figure S1. Photographs of (1) Liposome, (2) B-PDEAEA/TRAIL@LIPO, (3) IR780Liposome and (4) B-PDEAEA/TRAIL@IR780-LIPO dispersed in PBS.
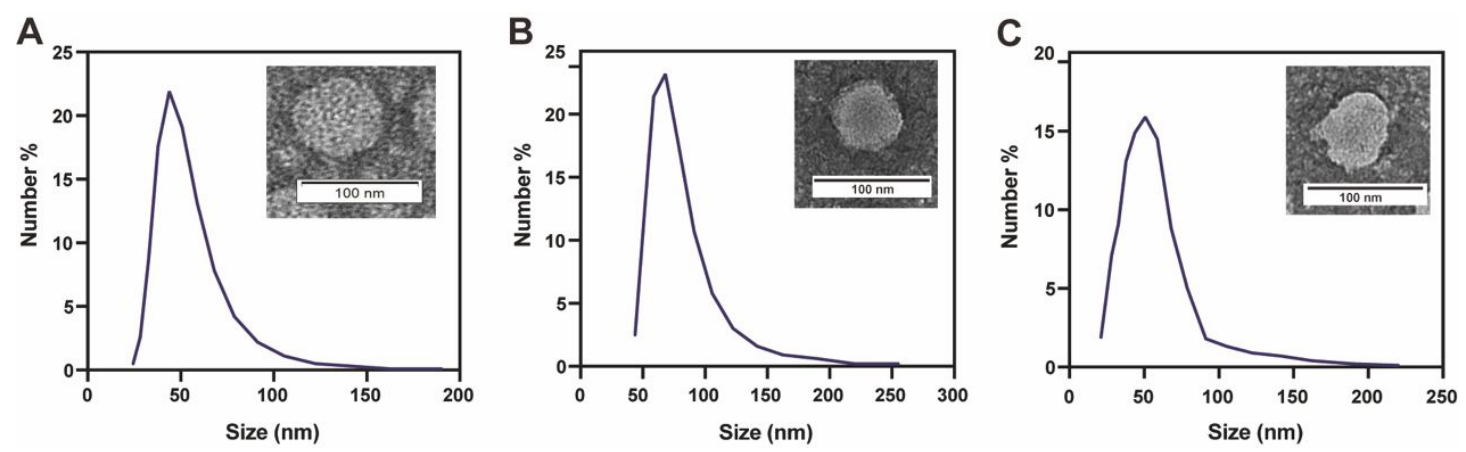
Figure S2. Representative size distribution measured by dynamic light scattering (DLS) and transmission electron microscope (TEM) images of (A) Lip, (B) BTL and (C) IL. The scale bar is $100 \mathrm{~nm}$.

A

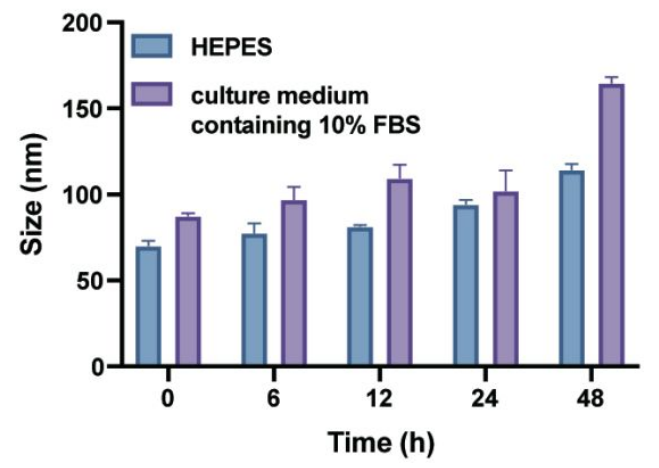

B

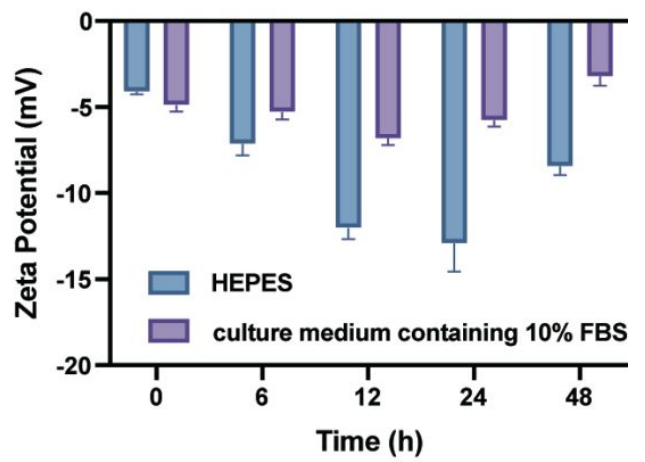

Figure S3. Changes of the size and zeta potential of BTIL lipopolyplexes after incubation in HEPES and culture medium containing 10\% FBS for $48 \mathrm{~h}$.

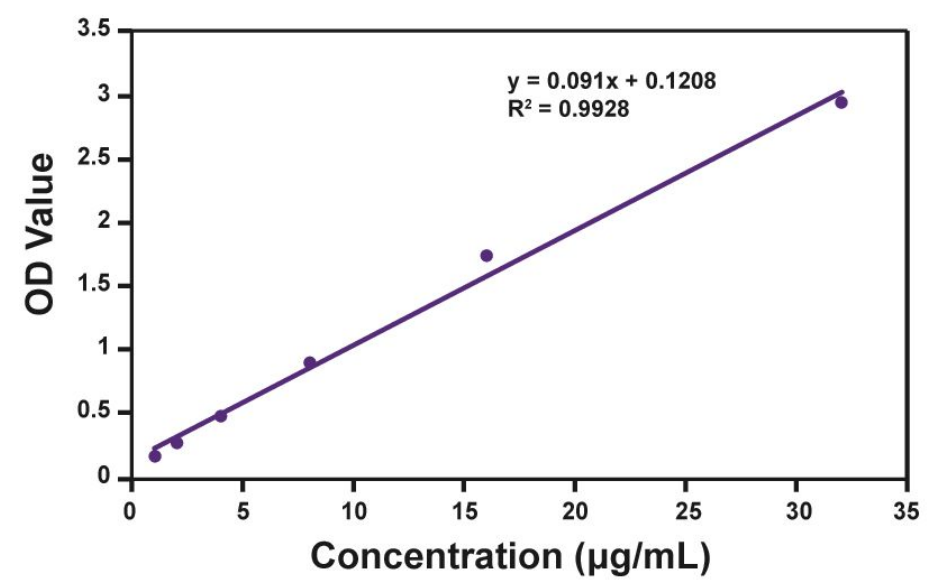

Figure S4. Standard curve of IR780 (concentration range: $0.976-31.25 \mu \mathrm{g} / \mathrm{mL}$ ). 

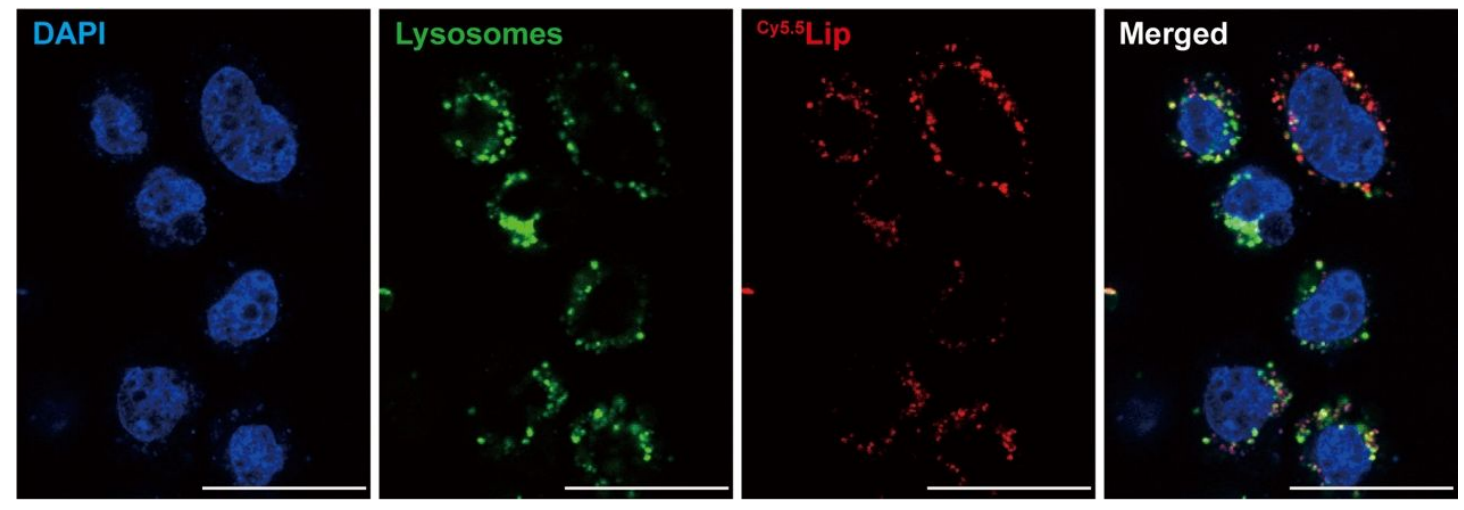

Figure S5. Confocal laser scanning microscopy images of BxPC-3 cells cultured with

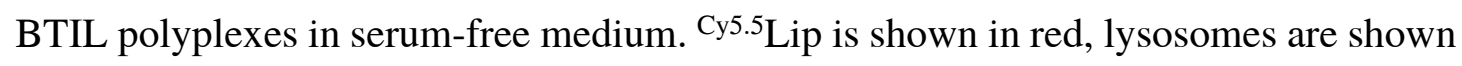
in green and the cell nuclei are shown in blue. All scale bars are $75 \mu \mathrm{m}$.

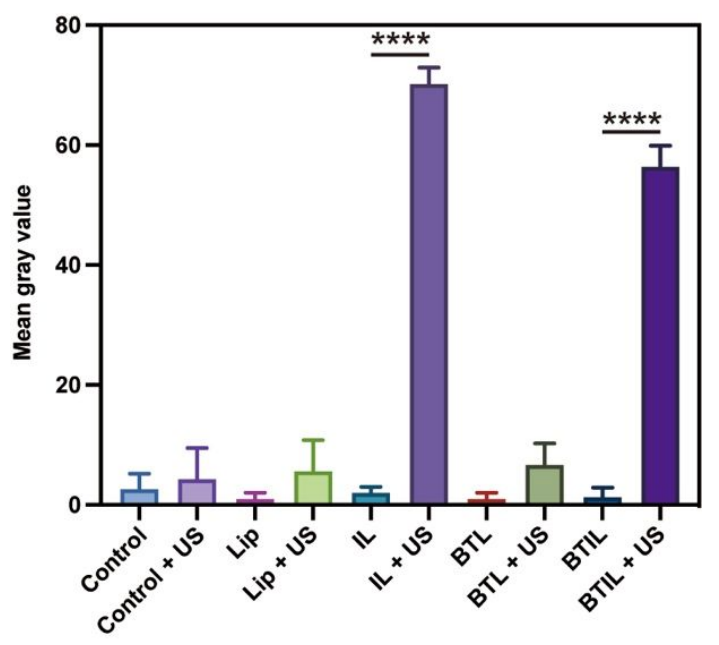

Figure S6. Corresponding mean fluorescent intensity of DCFH-DA analyzed by Image J software. ${ }^{* * * *} P<0.0001$. 


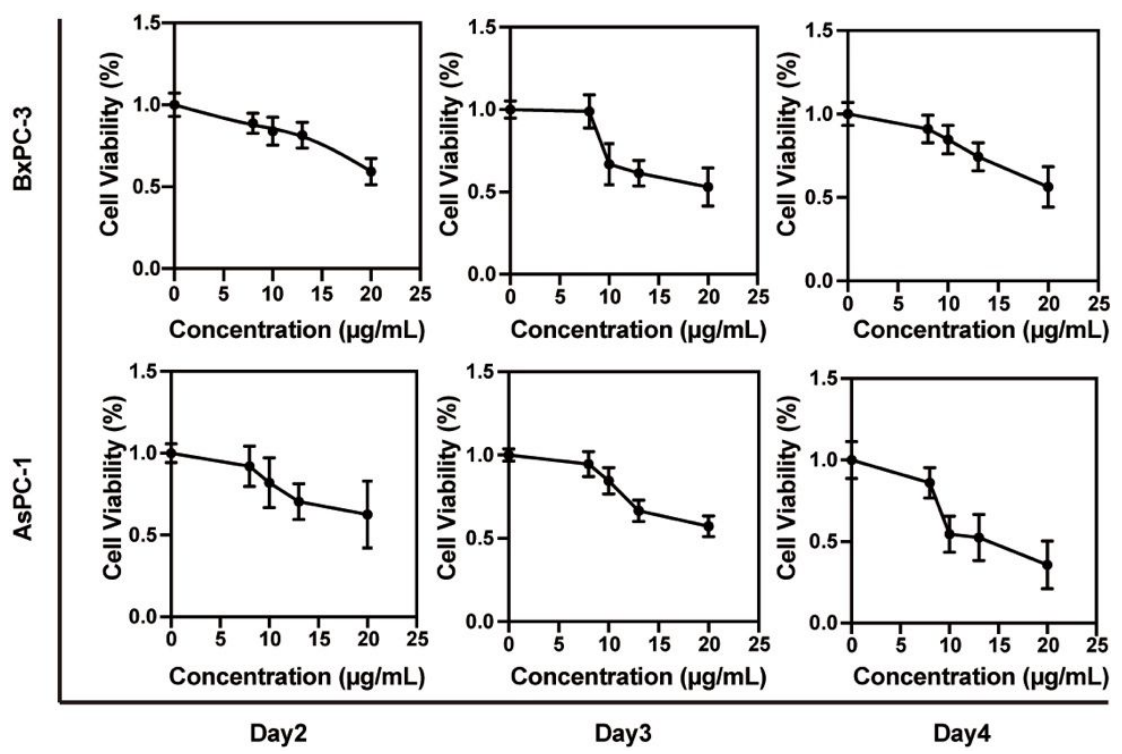

Figure S7. Cell viability of BxPC-3 and AsPC-1 cells incubated with BTL polyplexes at different concentrations for $48 \mathrm{~h}, 72 \mathrm{~h}$ and $96 \mathrm{~h}$.

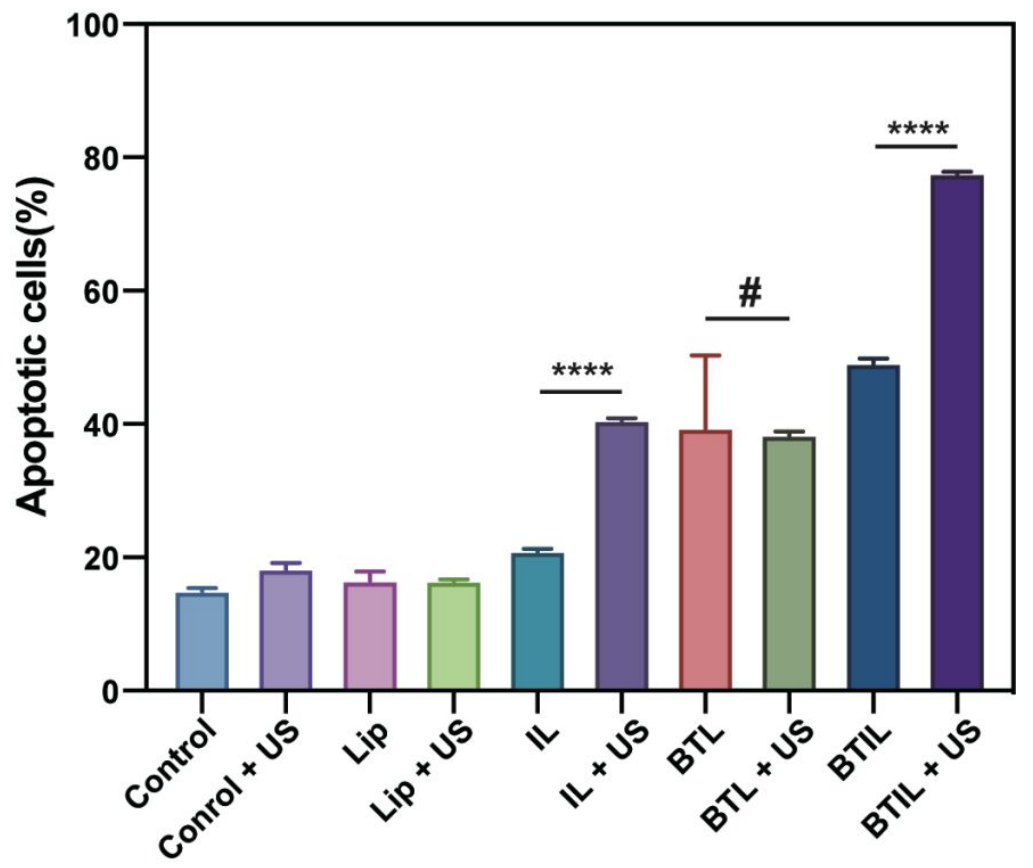

Figure S8. The percentage of apoptotic cells in different treatments. ${ }^{*} P<0.05,{ }^{* *} P<0.01$,

${ }^{* * *} P<0.001,{ }^{* * * *} P<0.0001$ and ${ }^{\#} P>0.05$. 


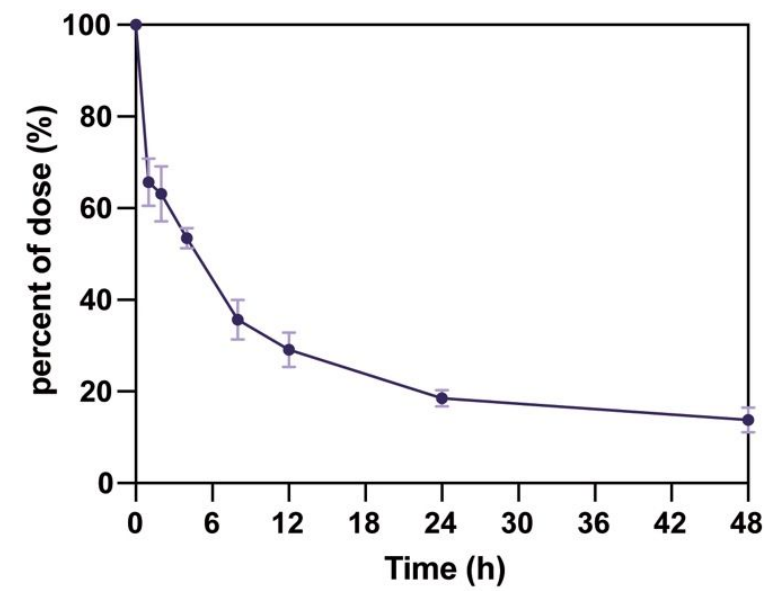

Figure S9. The blood clearance kinetics of BTIL polyplexes after intravenously administration at a TRAIL-equivalent dose of $1 \mathrm{mg} / \mathrm{kg}$.

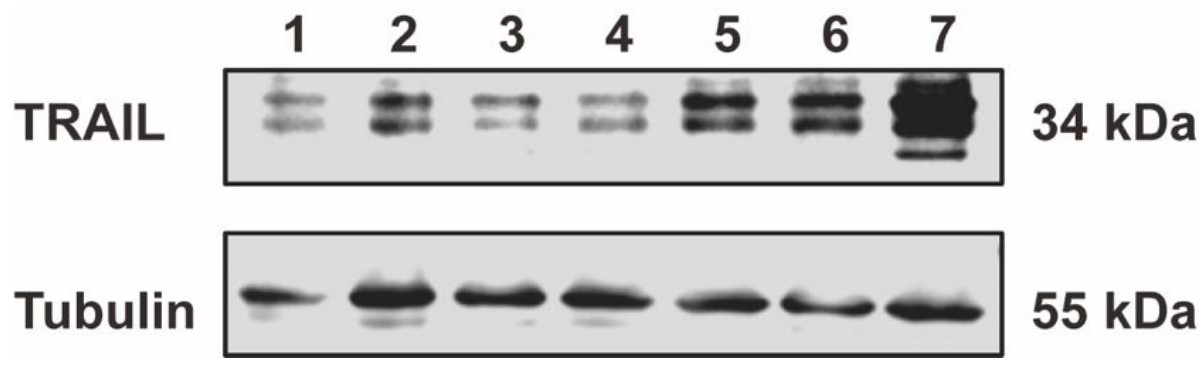

Figure S10. Western blot assay of TRAIL expression in tumors among various groups.

1: Control; 2: Control + US; 3: IL; 4: IL + US; 5: BTL; 6: BTIL; 7: BTIL + US. 


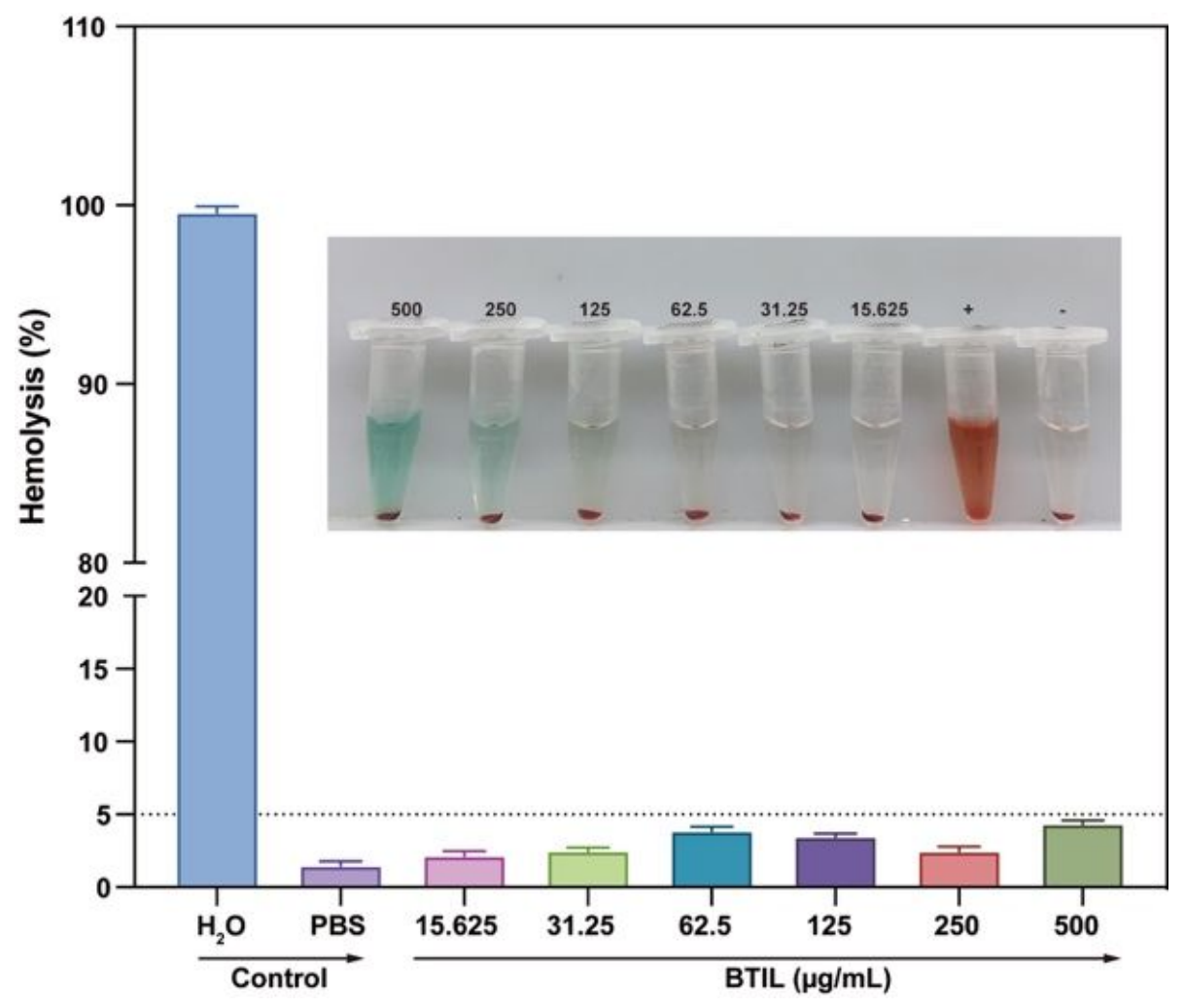

Figure S11. Hemolysis ratios of BTIL solutions with different concentrations treated with red blood cells, PBS as a negative control and deionized water as a positive control respectively. Inset: Hemolysis photographs after centrifugation. 

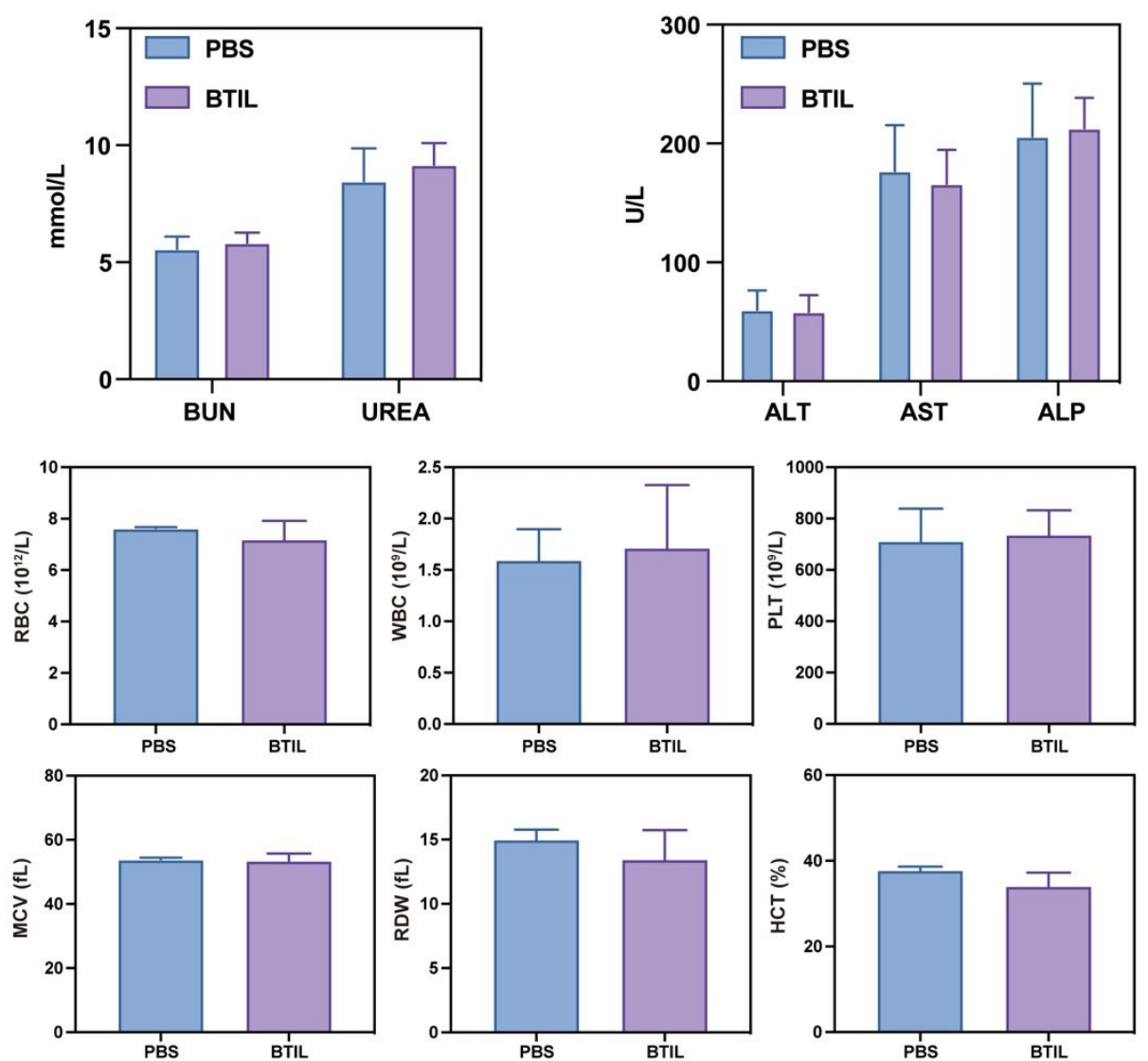

Figure S12. The blood cell analysis and the detection of blood biochemistry indicators in PBS and BTIL groups.

\section{REFERENCES}

(1) Liu, X.; Xiang, J.; Zhu, D.; Jiang, L.; Zhou, Z.; Tang, J.; Liu, X.; Huang, Y.; Shen,

Y. Fusogenic Reactive Oxygen Species Triggered Charge-Reversal Vector for Effective Gene Delivery. Advanced Materials 2015, 28 (9), 1743-1752.

(2) Elsana, H.; Olusanya, T. O. B.; Carr-Wilkinson, J.; Darby, S.; Faheem, A.; Elkordy, A. A. Evaluation of Novel Cationic Gene Based Liposomes with Cyclodextrin Prepared by Thin Film Hydration and Microfluidic Systems. Sci Rep 2019, 9 (1), 15120. 
S-1 